\title{
Parameterized Inapproximability of Target Set Selection and Generalizations
}

\author{
Cristina Bazgan \\ PSL, Université Paris-Dauphine, LAMSADE UMR CNRS 7243, France \\ Institut Universitaire de France \\ bazgan@lamsade.dauphine.fr \\ Morgan Chopin \\ Institut für Optimierung und Operations Research, Universität Ulm, Germany \\ morgan.chopin@uni-ulm.de \\ André Nichterlein \\ Institut für Softwaretechnik und Theoretische Informatik, TU Berlin, Germany \\ andre.nichterleinetu-berlin.de \\ Florian Sikora \\ PSL, Université Paris-Dauphine, LAMSADE UMR CNRS 7243, France \\ florian.sikora@lamsade.dauphine.fr
}

\begin{abstract}
In this paper, we consider the TARGET SET SELECTION problem: given a graph and a threshold value thr $(v)$ for each vertex $v$ of the graph, find a minimum size vertex-subset to "activate" such that all vertices of the graph are activated at the end of the propagation process. A vertex $v$ is activated during the propagation process if at least $\operatorname{thr}(v)$ of its neighbors are activated. This problem models several practical issues like faults in distributed networks or word-to-mouth recommendations in social networks. We show that for any functions $f$ and $\rho$ this problem cannot be approximated within a factor of $\rho(k)$ in $f(k) \cdot n^{O(1)}$ time, unless FPT $=\mathrm{W}[\mathrm{P}]$, even for restricted thresholds (namely constant and majority thresholds), where $k$ is the number of vertices to activate in the beginning. We also study the cardinality constraint maximization and minimization versions of the problem for which we prove similar hardness results.
\end{abstract}

\section{Introduction}

Diffusion processes in graphs have been intensively studied [1, 4, 6, 7, 14, 16, 21, 22]. One model to represent them is to define a propagation rule and choose a subset of vertices that, according to the given rule, activates all or a fixed fraction of the vertices where initially all but the chosen vertices are inactive. This models problems such as the spread of influence or information in social networks via word-of-mouth recommendations, of diseases in populations, or of faults in distributed computing [14, 16, 21]. One representative problem that appears in this context is the INFLUENCE MAXIMIZATION problem introduced by Kempe et al. [16]. Given a directed graph and an integer $k$, the task is to choose a vertex subset of size at most $k$ such that the number of activated vertices at the end of the propagation process is maximized. The authors show that the problem is polynomial-time $\left(\frac{e}{e-1}+\varepsilon\right)$ approximable for any $\varepsilon>0$ under some stochastic propagation rules, but NP-hard to approximate within a ratio of $n^{1-\varepsilon}$ for any $\varepsilon>0$ for general propagation rules.

In this paper, we use the following deterministic propagation model. We are given an undirected graph, a threshold value $\operatorname{thr}(v)$ associated to each vertex $v$, and the following propagation rule: a vertex becomes active if at

\footnotetext{
*An extended abstract was accepted for publication in the Proceedings of the 10th conference Computability in Europe (CiE 2014)
} 
least thr $(v)$ many neighbors of $v$ are active. The propagation process proceeds in several rounds and stops when no further vertex becomes active. Given this model, finding and activating a minimum-size vertex subset such that all the vertices become active is known as the TARGET SET SELECTION problem and was introduced by Chen [7].

TARGET SET SELECTION has been shown NP-hard even for bipartite graphs of bounded degree when all thresholds are at most two [7]. Moreover, the problem was shown to be hard to approximate in polynomial time within a ratio $O\left(2^{\log ^{1-\varepsilon} n}\right)$ for any $\varepsilon>0$, even for constant degree graphs with thresholds at most two and for general graphs when the threshold of each vertex is half its degree (called majority thresholds) [7]. If the threshold of each vertex equals its degree (unanimity thresholds), then the problem is equivalent to the vertex cover problem [7] and, thus, admits a 2-approximation and is hard to approximate with a ratio better than 1.36 [11]. Concerning the parameterized complexity, the problem is known to be W[2]-hard with respect to (w.r.t.) the solution size, even on bipartite graphs of diameter four with majority thresholds or thresholds at most two [19]. Furthermore, it is W[1]-hard w.r.t. each of the parameters "treewidth", "cluster vertex deletion number", and "pathwidth" [4, 9]. On the positive side, the problem becomes fixed-parameter tractable w.r.t. each of the single parameters "vertex cover number", "feedback edge set size", and "bandwidth" [9, 19]. If the input graph is complete, has a bounded cliquewidth, or has a bounded treewidth and bounded thresholds then the problem is polynomial-time solvable [4, 10, 19].

Motivated by the hardness of approximation and parameterized hardness we showed in previous work [3] that the cardinality constraint maximization version of TARGET SET SELECTION, that is to find a fixed number $k$ of vertices to activate such that the number of activated vertices at the end is maximum, is strongly inapproximable in fpt-time w.r.t. the parameter $k$, even for restricted thresholds. For the special case of unanimity thresholds, we showed that the problem is still inapproximable in polynomial time, but becomes $r(n)$-approximable in fpt-time w.r.t. the parameter $k$, for any strictly increasing function $r$.

Continuing this line of research, we study in this paper TARGET SET SELECTION and its variants where the parameter relates to the optimum value. This requires the special definition of "fpt cost approximation" since in parameterized problems the parameter is given which is not the case in optimization problems (see Section 2 for definitions). Fpt approximation algorithms were introduced in [5, 8, 13], see also the survey of Marx [17]. Besides this technical difference observe that TARGET SET SELECTION can be seen as a special case of the previously considered problem, since activating all vertices is a special case of activating a given number of vertices. Strengthening the known inapproximability results, we first prove in Section 3 that TARGET SET SELECTION is not fpt cost $\rho$-approximable, for any computable function $\rho$, unless FPT $=\mathrm{W}[\mathrm{P}]$, even for majority and constant thresholds. Complementing our previous work, we also study in Section 4 the cardinality constraint maximization and minimization versions of TARGET SET SELECTION. We prove that these two problems are not fpt cost $\rho$-approximable, for any computable function $\rho$, unless FPT $=\mathrm{W}[1]$. Note that we study the parameterized approximability of these problems in a different way as in our previous work [3]. Indeed, here, the parameter is related to the solution size (i.e. the number of vertices activated in the end), while in our previous work, the parameter was the size of the set of vertices initially activated.

\section{Preliminaries and basic observations}

In this section, we provide basic backgrounds and notation used throughout this paper and define TARGET SET SELECTION. For details on parameterized complexity we refer to the monographs [12, 15, 20]. For details on parameterized approximability we refer to the survey of Marx [17].

Graph terminology. Let $G=(V, E)$ be an undirected graph. For a subset $S \subseteq V, G[S]$ is the subgraph induced by $S$. The open neighborhood of a vertex $v \in V$ in $G$, denoted by $N_{G}(v)$, is the set of all neighbors of $v$ in $G$. The closed neighborhood of a vertex $v$ in $G$, denoted $N_{G}[v]$, is the set $N_{G}(v) \cup\{v\}$. The degree of a vertex $v$ is denoted by $\operatorname{deg}_{G}(v)$ and the maximum degree of the graph $G$ is denoted by $\Delta_{G}$. We skip the subscripts if $G$ is clear from the context.

Parameterized complexity. A parameterized problem $(I, k)$ is said fixed-parameter tractable (or in the class FPT) w.r.t. parameter $k$ if it can be solved in $f(k) \cdot|I|^{c}$ time, where $f$ is any computable function and $c$ is a constant. The 
parameterized complexity hierarchy is composed of the classes FPT $\subseteq \mathrm{W}[1] \subseteq \mathrm{W}[2] \subseteq \cdots \subseteq \mathrm{W}[\mathrm{P}] . \mathrm{A}$ W[1]-hard problem is not fixed-parameter tractable (unless FPT $=\mathrm{W}[1]$ ) and one can prove the W[1]-hardness by means of a parameterized reduction from a W[1]-hard problem. Such a reduction between two parameterized problems $A_{1}$ and $A_{2}$ is a mapping of any instance $(I, k)$ of $A_{1}$ in $g(k) \cdot|I|^{O(1)}$ time (for some computable function $g$ ) into an instance $\left(I^{\prime}, k^{\prime}\right)$ for $A_{2}$ such that $(I, k) \in A_{1} \Leftrightarrow\left(I^{\prime}, k^{\prime}\right) \in A_{2}$ and $k^{\prime} \leq h(k)$ for some function $h$.

Parameterized approximation. An NP-optimization problem $Q$ is a tuple $(\mathcal{I}, S o l$, val, goal $)$, where $\mathcal{I}$ is the set of instances, $\operatorname{Sol}(I)$ is the set of feasible solutions for instance $I, \operatorname{val}(I, S)$ is the value of a feasible solution $S$ of $I$, and goal is either max or min. We assume that $\operatorname{val}(I, S)$ is computable in polynomial time and that $|S|$ is polynomially bounded by $|I|$ i.e. $|S| \leq|I|^{O(1)}$.

Definition 2.1 (fpt cost $\boldsymbol{\rho}$-approximation algorithm, Chen et al. [8]). Let $Q$ be an optimization problem and $\rho: \mathbb{N} \rightarrow \mathbb{R}$ be a function such that $\rho(k) \geq 1$ for every $k \geq 1$ and $k \cdot \rho(k)$ is nondecreasing (when goal = min) or $\frac{k}{\rho(k)}$ is unbounded and nondecreasing (when goal $=\max$ ). A decision algorithm $\mathcal{A}$ is an fpt cost $\rho$-approximation algorithm for $Q$ (when $\rho$ satisfies the previous conditions) if for every instance $I$ of $Q$ and integer $k$, with $\operatorname{Sol}(I) \neq \emptyset$, its output satisfies the following conditions:

1. If $\operatorname{opt}(I)>k($ when goal $=\min )$ or $\operatorname{opt}(I)<k($ when goal $=\max )$, then $\mathcal{A}$ rejects $(I, k)$.

2. If $k \geq \operatorname{opt}(I) \cdot \rho(\operatorname{opt}(I))($ when $\operatorname{goal}=\min )$ or $k \leq \frac{\operatorname{opt}(I)}{\rho(\operatorname{opt}(I))}($ when $g o a l=\max )$, then $\mathcal{A}$ accepts $(I, k)$.

Moreover the running time of $\mathcal{A}$ on input $(I, k)$ is $f(k) \cdot|I|^{O(1)}$. If such a decision algorithm $\mathcal{A}$ exists then $Q$ is called fpt cost $\rho$-approximable.

The notion of a gap-reduction was introduced in [2] by Arora and Lund. We use in this paper a variant of this notion, called fpt gap-reduction.

Definition 2.2 (fpt gap-reduction). A problem $A$ parameterized by $k$ is called fpt gap-reducible to an optimization problem $Q$ with gap $\rho$ if for any instance $(I, k)$ of $A$ we can construct an instance $I^{\prime}$ of $Q$ in $f(k) \cdot|I|^{O(1)}$ time while satisfying the following properties:

1. If $I$ is a yes instance then $\operatorname{opt}\left(I^{\prime}\right) \leq \frac{g(k)}{\rho\left(\operatorname{opt}\left(I^{\prime}\right)\right)}($ when goal $=\min )$ or $\operatorname{opt}\left(I^{\prime}\right) \geq g(k) \rho\left(\operatorname{opt}\left(I^{\prime}\right)\right)($ when goal $=$ $\max$ ),

2. If $I$ is a no instance then $\operatorname{opt}\left(I^{\prime}\right)>g(k)($ when goal $=\min )$ or $\operatorname{opt}\left(I^{\prime}\right)<g(k)($ when goal $=\max )$,

for some function $g$. The function $\rho$ satisfies the aforementioned conditions.

The interest of the fpt gap-reduction is the following result that immediately follows from the previous definition:

Lemma 2.3. If a parameterized problem $A$ is $\mathcal{C}$-hard and fpt gap-reducible to an optimization problem $Q$ with gap $\rho$ then $Q$ is not fpt cost $\rho$-approximable unless $\mathrm{FPT}=\mathcal{C}$ where $\mathcal{C}$ is any class of the parameterized complexity hierarchy.

Problem statement. Let $G=(V, E)$ be an undirected graph and let thr: $V \rightarrow \mathbb{N}$ be a threshold function such that $1 \leq \operatorname{thr}(v) \leq \operatorname{deg}(v)$, for all $v \in V$. The definition of TARGET SET SELECTION is based on the notion of "activation". Let $S \subseteq V$. Informally speaking, a vertex $v \in V$ gets activated by $S$ in the $i^{\text {th }}$ round if at least $\operatorname{thr}(v)$ of its neighbors are active after the previous round (where $S$ are the vertices active in the $0^{\text {th }}$ round). Formally, for a vertex set $S$, let $\mathcal{A}_{G, \text { thr }}^{i}(S)$ denote the set of vertices of $G$ that are activated by $S$ at the $i^{\text {th }}$ round, with

$$
\begin{aligned}
& \mathcal{A}_{G, \mathrm{thr}}^{0}(S)=S \text { and } \\
& \mathcal{A}_{G, \mathrm{thr}}^{i+1}(S)=\mathcal{A}_{G, \mathrm{thr}}^{i}(S) \cup\left\{v \in V:\left|N(v) \cap \mathcal{A}_{G, \mathrm{thr}}^{i}(S)\right| \geq \operatorname{thr}(v)\right\} .
\end{aligned}
$$

For $S \subseteq V$, the unique positive integer $r$ with $\mathcal{A}_{G, \mathrm{thr}}^{r-1}(S) \neq \mathcal{A}_{G \text {,thr }}^{r}(S)=\mathcal{A}_{G, \text { thr }}^{r+1}(S)$ is called the number $r_{G}(S)$ of activation rounds. It is easy to see that $r_{G}(S) \leq|V(G)|$ for all graphs $G$. Furthermore, we call $\mathcal{A}_{G \text {, thr }}(S)=\mathcal{A}_{G \text {,thr }}^{r_{G}(S)}(S)$ the 3 


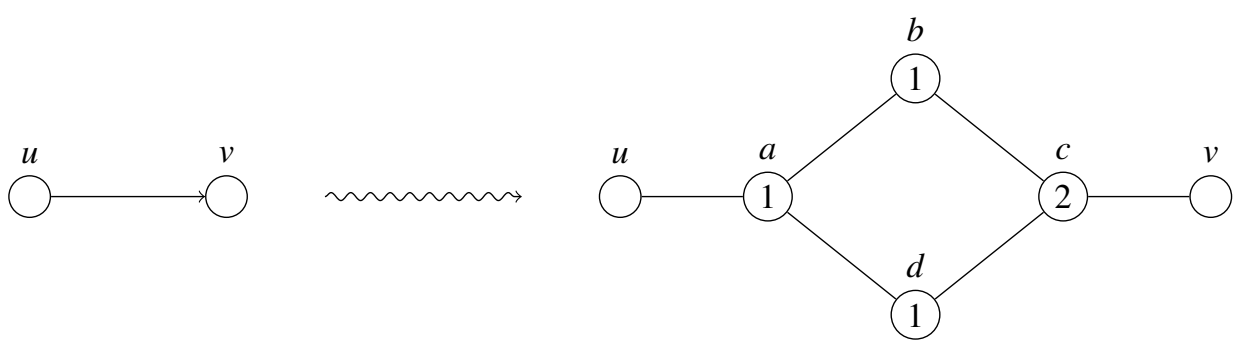

Figure 1. Illustration of the directed edge gadget from $u$ to $v$.

set of vertices that are activated by $S$. If $\mathcal{A}_{G, \mathrm{thr}}(S)=V$, then $S$ is called a target set for $G$. TARGET SET SELECTION is formally defined as follows.

\author{
TARGET SET SELECTION \\ Input: A graph $G=(V, E)$ and a threshold function thr: $V \rightarrow \mathbb{N}$. \\ Output: A target set for $G$ of minimum cardinality.
}

We also consider the following cardinality constrained version.

\title{
MAX Closed $k$-INFLUENCE
}

Input: A graph $G=(V, E)$, a threshold function thr: $V \rightarrow \mathbb{N}$, and an integer $k$.

Output: A subset $S \subseteq V$ with $|S| \leq k$ maximizing $\left|\mathcal{A}_{G \text {,thr }}(S)\right|$.

The MAX OPEn $k$-INFLUENCE problem asks for a set $S \subseteq V$ with $|S| \leq k$ such that $\left|\mathcal{A}_{G \text {,thr }}(S) \backslash S\right|$ is maximum. We remark that this difference in the definition is important when considering the approximability of these problems. Finally, Min Closed $k$-Influence (resp. Min Open $k$-InfluenCE) is also defined similarly, but one ask for a solution $S \subseteq V$ with $|S|=k$ such that $\left|\mathcal{A}_{G \text {,thr }}(S)\right|$ is minimum (resp. $\left|\mathcal{A}_{G \text {,thr }}(S) \backslash S\right|$ is minimum).

Directed edge gadget. We will use the directed edge gadget as used and proved by Chen [7] throughout our work: A directed edge gadget from a vertex $u$ to another vertex $v$ consists of a 4-cycle $\{a, b, c, d\}$ such that $a$ and $u$ as well as $c$ and $v$ are adjacent. Moreover $\operatorname{thr}(a)=\operatorname{thr}(b)=\operatorname{thr}(d)=1$ and $\operatorname{thr}(c)=2$ (see Figure 1). The idea is that the vertices in the directed edge gadget become active if $u$ is activated but not if $v$ is activated. Hence, the activation process may go from $u$ to $v$ via the gadget but not in the reverse direction.

\section{Parameterized inapproximability of TARGET SET SELECTION}

Marx [18] showed that the Monotone CiRCuit SATISFIABILITy problem admits no fpt cost $\rho$-approximation algorithm for any function $\rho$ unless FPT $=\mathrm{W}[\mathrm{P}]$. In this section we show that we can transfer this strong inapproximability result from Monotone Circuit SATisfiability to TARGET SET SElection.

Before defining Monotone Circuit SATisfiability, we recall the following notations. A monotone (boolean) circuit is a directed acyclic graph. The nodes with in-degree at least two are labeled with and or with $o r$, the $n$ nodes with in-degree zero are input nodes, and due to the monotonicity there are no nodes with in-degree one (negation nodes in standard circuits). Furthermore, there is one node with out-degree zero, called the output node. For an assignment of the input nodes with true/false, the circuit is satisfied if the output node is evaluated (in the natural way) to true. The weight of an assignment is the number of input nodes assigned to true. We denote an assignment as a set $A \subseteq\{1, \ldots, n\}$ where $i \in A$ if and only if the $i^{\text {th }}$ input node is assigned to true. The MonOTONE CIRCUIT SATISFIABILITY problem is then defined as follows:

MONOTONE CIRCUIT SATISFIABILITY

Input: A monotone circuit $C$.

Output: A satisfying assignment of minimum weight, that is, a satisfying assignment with a minimum number of input nodes set to true. 

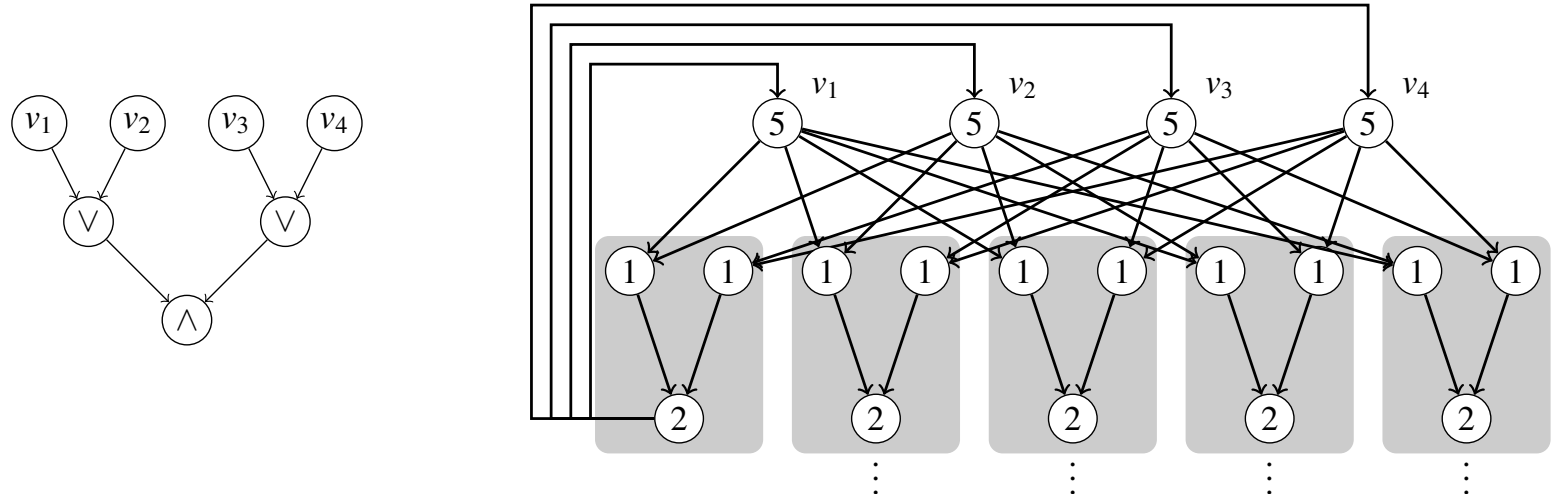

Figure 2. Illustration of the reduction described in Theorem 3.1. All arrows on the right graph represent directed edge gadgets. Thresholds are displayed inside each vertex.

By reducing Monotone Circuit Satisfiability to Target Set Selection in polynomial time such that there is a "one-to-one" correspondence between the solutions, we show the inapproximability result transfers to TARget Set Selection. First, we show one reduction working with general thresholds. Using further gadgets, we describe how to achieve constant or majority thresholds in our constructed instance.

\subsection{General thresholds}

As mentioned above, we will reduce from Monotone CirCUit SatisfiabiLity, and thus derive the same inapproximability result for TARget Set Selection as for Monotone Circuit Satisfiability.

Theorem 3.1. Target Set Selection is not fpt cost $\rho$-approximable, for any computable function $\rho$, unless $\mathrm{FPT}=\mathrm{W}[\mathrm{P}]$.

Proof. Let $C$ be an instance of Monotone Circuit Satisfiability. We construct an instance of Target Set SELECTION as follows. Initialize $G=(V, E)$ as a copy of the directed acyclic graph $C$ where each directed edge is replaced by a directed edge gadget. We call a vertex in $G$ an input vertex (resp. output vertex, and-vertex, or-vertex) if it corresponds to an input node (resp. output node, and-node, or-node). Next, for each and-node in $C$ with in-degree $d$ set the threshold of the corresponding and-vertex in $G$ to $d$ and for each or-vertex in $G$ set the threshold to 1 . Set the threshold of each input vertex in $G$ to $n+1$. Next, add $n$ copies to $G$ and "merge" all vertices corresponding to the same input node. This means, that for an input node $v$ with an outgoing edge $(v, w)$ in $C$ the graph $G$ contains $n+1$ vertices $w_{1}, \ldots, w_{n+1}$ and $n+1$ directed edges from $v$ to $w_{i}, 1 \leq i \leq n+1$. Finally, add directed edges from each output vertex to each input vertex. This completes our construction (see Figure 2).To complete the proof, it remains to show that

(i) for every satisfying assignment $A$ for $C$ there exists a target set of size $|A|$ for $G$, and

(ii) for every target set $S$ for $G$ there exists a satisfying assignment of size $|S|$ for $C$.

(i) Let $A \subseteq\{1, \ldots, n\}$ be a satisfying assignment for $C$. We show that the set $S$ of vertices of $G$ that correspond to the input nodes in $A$ forms a target set. Clearly, $|S|=|A|$. First, observe that by construction, all the $n+1$ output vertices of $G$ become active. Hence, also all input vertices that are not in $S$ become active. Thus, all remaining vertices in $G$ are activated since $\operatorname{thr}(v) \leq \operatorname{deg}(v)$ for all $v \in V$.

(ii) Let $S \subseteq V$ be a target set for $G$. First, observe that we can assume that $|S|<n$ since otherwise the satisfying assignment simply sets all input nodes to true. Next, observe that we can assume that $S$ is a subset of the input vertices. Indeed, since $G$ contains $n+1$ copies of the circuit (excluding the input vertices), there is at least one copy without vertices in $S$ and, hence, the output vertex in that copy becomes active solely because of the input vertices in $S$. Finally, assume by contradiction that the set of input nodes that correspond to the vertices in $S$ does not 5 


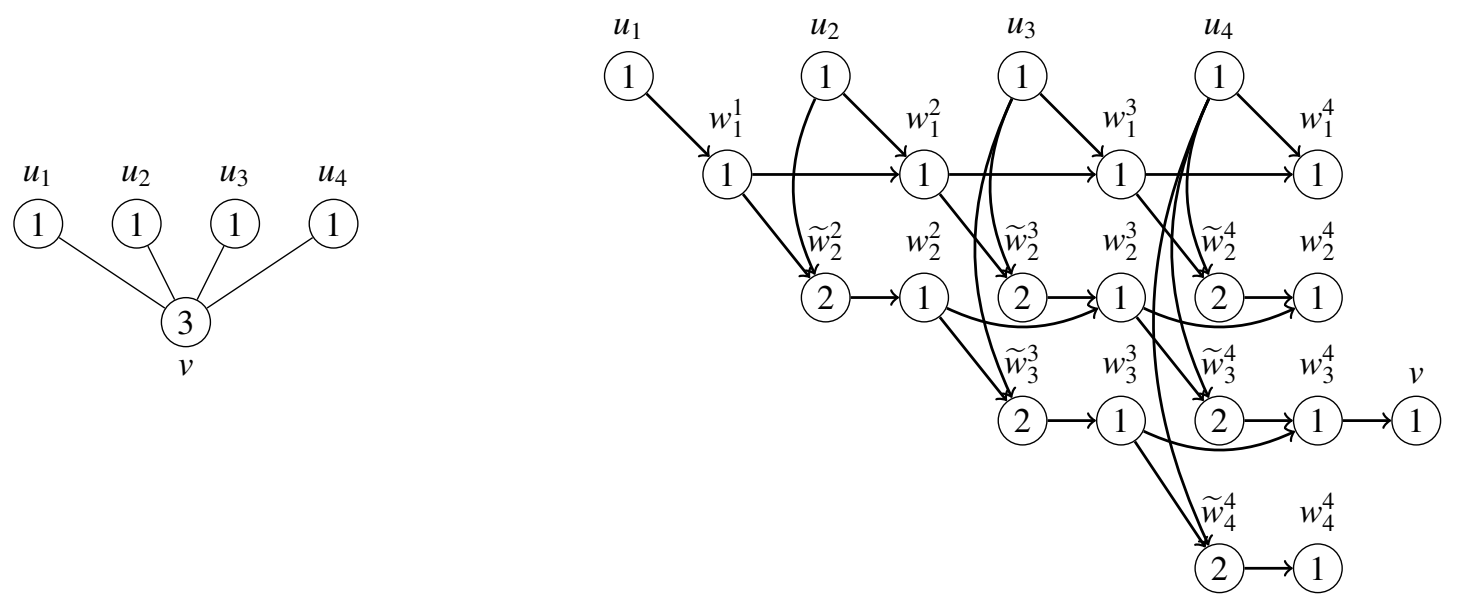

Figure 3. Example of an activation gadget $g_{v}$ for a vertex $v$ with $\operatorname{deg}(v)=4$ and $\operatorname{thr}(v)=3$. All arrows on the right graph are directed edge gadgets. Thresholds are represented inside each vertex.

form a satisfying assignment. Hence, the output node of $C$ is evaluated to false. However, due to the construction, this implies that the vertices corresponding to the output node are not activated, contradicting that $S$ is a target set for $G$.

\subsection{Restricted thresholds}

In this subsection, we enhance the inapproximability results to variants of TARGET SET SELECTION with restricted threshold functions. To this end, we use the construction desbribed in Lemma 2 of [19] which transforms in polynomial time any instance $I=(G=(V, E)$, thr $)$ of TARGET SET SELECTION into a new instance $I^{\prime}=\left(G^{\prime}=\right.$ $\left.\left(V^{\prime}, E^{\prime}\right), t h r^{\prime}\right)$ where $\operatorname{thr}^{\prime}$ is the majority function such that

(i) for every target set $S$ for $I$ there is a target set $S^{\prime}$ for $I^{\prime}$ with $\left|S^{\prime}\right| \leq|S|+1$, and

(ii) for every target set $S^{\prime}$ for $I^{\prime}$ there is a target set $S$ for $I$ with $|S| \leq\left|S^{\prime}\right|-1$.

Hence, the next corollary follows.

Corollary 3.2. TARGET SET SELECTION with majority thresholds is not fpt cost $\rho$-approximable, for any computable function $\rho$, unless $\mathrm{FPT}=\mathrm{W}[\mathrm{P}]$.

Next, we show a similar statement for constant thresholds.

Lemma 3.3. Let $I=(G=(V, E)$, thr $)$ be an instance of TARGET SET SELECTION. Then, we can construct in polynomial time an instance $I^{\prime}=\left(G^{\prime}=\left(V^{\prime}, E^{\prime}\right)\right.$, thr $\left.^{\prime}\right)$ of TARGET SET SELECTION where $\operatorname{thr}^{\prime}(v) \leq 2$ for all $v \in$ $V^{\prime}$ and $G^{\prime}$ is bipartite such that

(i) for every target set $S$ for I there is a target set $S^{\prime}$ for $I^{\prime}$ with $\left|S^{\prime}\right|=|S|$, and

(ii) for every target set $S^{\prime}$ for $I^{\prime}$ there is a target set $S$ for I with $|S| \leq\left|S^{\prime}\right|$.

Proof. Let $I=(G=(V, E)$, thr $)$ be an instance of TARget Set Selection. We construct $I^{\prime}=\left(G^{\prime}=\right.$ $\left(V^{\prime}, E^{\prime}\right)$, thr $\left.^{\prime}\right)$ as follows: Start by copying $V$ into $V^{\prime}$, and set $\operatorname{thr}^{\prime}(v)=\operatorname{thr}(v)$ for all $v \in V$. For each vertex $v \in V^{\prime}$ with $\operatorname{thr}^{\prime}(v) \leq 2$ add for each $u \in N_{G}(v)$ a directed edge gadget from $u$ to $v$. Then, for each vertex $v \in V^{\prime}$ with $\operatorname{thr}^{\prime}(v)>2$ we add an activation gadget $g_{v}$ that activates $v$ if and only if at least $\operatorname{thr}(v)$ neighbors of $v$ are active (see Figure 3). Let $N_{G}(v)=\left\{u_{1}, \ldots, u_{d}\right\}$ be the neighbors of $v$ in $G$. Add the vertices $w_{j}^{i}, 1 \leq j \leq i \leq d$ where the vertex $w_{j}^{i}, j \leq i$, will become active if at least $j$ vertices from $\left\{u_{1}, \ldots, u_{i}\right\}$ are active. We inductively describe how to complete the gadget: First, set $\operatorname{thr}\left(w_{1}^{1}\right)=1$ and add a directed edge gadget from $u_{1}$ to $w_{1}^{1}$. Now, 
let $i \in\{2, \ldots, d\}$. Then, set $\operatorname{thr}\left(w_{1}^{i}\right)=1$ and add two directed edge gadgets to $w_{1}^{i}$, one from $u_{i}$ and one from $w_{1}^{i-1}$. For $j \in\{2, \ldots, i\}$, add a vertex $\widetilde{w}_{j}^{i}$ with threshold two and add two directed edge gadgets to $\widetilde{w}_{j}^{i}$, one from $u_{i}$ and one from $w_{j-1}^{i-1}$. Next, set $\operatorname{thr}\left(w_{j}^{i}\right)=1$ and add a directed edge gadget from $\widetilde{w}_{j}^{i}$ to $w_{j}^{i}$ and if $j<i$, then add one directed edge gadget from $w_{j}^{i-1}$ to $w_{j}^{i}$. Observe that $w_{j}^{i}$ becomes active if and only if $u_{i}$ and $j-1$ vertices from $\left\{u_{1}, \ldots, u_{i-1}\right\}$ are active ( $\widetilde{w}_{j}^{i}$ is active in this case) or if $j$ vertices from $\left\{u_{1}, \ldots, u_{i-1}\right\}$ are active ( $w_{j}^{i-1}$ is active in this case). Finally, add a directed edge from $w_{\operatorname{thr}(v)}^{d}$ to $v$ and set $\operatorname{thr}(v)=1$. This completes the reduction. Clearly, $I^{\prime}$ can be computed in polynomial time and $\operatorname{thr}^{\prime}(v) \leq 2$ for all $v \in V^{\prime}$. Furthermore, observe that $G^{\prime}$ is bipartite. Indeed, after removing all vertices of directed edge gadgets from $G^{\prime}$ the resulting graph contains no edge. Furthermore, if there is a directed edge gadget from $u \in V^{\prime}$ to $v \in V^{\prime}$, then $u$ and $v$ are at distance four in $G^{\prime}$. Hence, all cycles in $G^{\prime}$ have an even length and, thus, $G^{\prime}$ is bipartite.

(i) Let $S \subseteq V$ be a target set for $G$. We show that $S \subseteq V^{\prime}$ is also a target set for $G^{\prime}$. Assume by contradiction that $S$ is not a target set for $G^{\prime}$, that is, there is at least one vertex in $G^{\prime}$ that is not activated by $S$. Denote by $X$ the set of all these vertices, $X=V^{\prime} \backslash \mathcal{A}_{G^{\prime}, \text { thr }^{\prime}}(S)$. Then let $v \in X$ be one of the earliest activated vertices in $G$, that is, $v \in \mathcal{A}_{G, \text { thr }}^{r}(S) \backslash \mathcal{A}_{G \text {,thr }}^{r-1}(S)$ for some $r \geq 1$ and $X \cap \mathcal{A}_{G \text {,thr }}^{r-1}(S)=\emptyset$. Hence, the set $Y=N_{G}(v) \cap \mathcal{A}_{G \text {,thr }}^{r-1}(S)$ has $|Y| \geq$ $\operatorname{thr}(v)$. By the choice of $v$ it follows that all vertices in $Y$ become active through $S, Y \subseteq \mathcal{A}_{G^{\prime}}, \operatorname{thr}^{\prime}(S)$. If $\operatorname{thr}(v) \leq 2$, then $v \in \mathcal{A}_{G^{\prime}, \text { thr }^{\prime}}(S)$, a contradiction. If $\operatorname{thr}(v)>2$, then by the construction of the activation gadget $g_{v}$, the vertex $w_{\operatorname{thr}(v)}^{\left|N_{G}(v)\right|}$ in $g_{v}$ becomes active. Thus $v \in \mathcal{A}_{G^{\prime}, \text { thr' }}(S)$, a contradiction. This implies that $V \subseteq \mathcal{A}_{G^{\prime}, \text { thr' }}(S)$. Furthermore, the set $V$ is clearly a target set for $G^{\prime}$, that is $\mathcal{A}_{G^{\prime}, \text { thr }}(V)=V^{\prime}$, and, hence, $S$ is a target set for $G^{\prime}$.

(ii) Let $S^{\prime}$ be a target set for $G^{\prime}$. Starting from $S^{\prime}$, we construct a target set $S \subseteq V$ for $G$ as follows: First, set $S=S^{\prime}$. Next, for each $v \in S \backslash V$ remove $v$ from $S$ and do the following: If $v$ is a vertex in a directed edge gadget from $u$ to $w$, then add $u$ to $S$. If $v$ is a vertex in an activation gadget $g_{u}$, then add $u$ to $S$. After exhaustively applying this procedure, we clearly have $S \subseteq V$. (When a vertex $v \in S \backslash V$ is replaced by a vertex $w$ contained in an activation gadget $g_{u}$, then $w$ will be replaced by $u$.) Next, we show that $S$ is a target set for $G$. Let $X=$ $V \backslash \mathcal{A}_{G, \mathrm{thr}}(S)$. Assume by contradiction that $X \neq \emptyset$. Then, let $v \in X$ be one of the "first non-activated" vertices in $X$, that is, $v \in \mathcal{A}_{G^{\prime} \text {,thr' }}^{r}\left(S^{\prime}\right) \backslash \mathcal{A}_{G^{\prime} \text {,thr' }}^{r-1}\left(S^{\prime}\right)$ for some $r \geq 1$ and $X \cap \mathcal{A}_{G^{\prime} \text {,thr' }}^{r-1}\left(S^{\prime}\right)=\emptyset$. Since $S^{\prime}$ is a target set for $G^{\prime}$, we have $v \in \mathcal{A}_{G^{\prime}, \text { thr' }}\left(S^{\prime}\right)$ and, due to the construction of $S, v \notin S^{\prime}$. Let $r^{\prime} \geq 1$ be the integer such that $v \in \mathcal{A}_{G^{\prime}, \text { thr' }}^{r^{\prime}}\left(S^{\prime}\right) \backslash$ $\mathcal{A}_{G^{\prime}, \text { thr }}^{r^{\prime}-1}\left(S^{\prime}\right)$. Let $Y=N_{G}(v) \cap \mathcal{A}_{G^{\prime}, \text { thr' }}^{r^{\prime}-1}\left(S^{\prime}\right)$. Due to the construction of $S$ and since $v$ is activated at time step $r^{\prime}$ in $G^{\prime}$, we know that there are at least thr $(v)$ activated vertices among $N(v)$ in $G^{\prime}$ at step $r^{\prime}-1$ (using the property of an activation gadget). It follows from the choice of $v$ that $Y \subseteq \mathcal{A}_{G, \text { thr }}(S)$, and hence $v \in \mathcal{A}_{G \text {, thr }}(S)$, a contradiction. Hence, $S^{\prime}$ is a target set for $G^{\prime}$ and by construction of $S$ it is clear that $|S| \leq\left|S^{\prime}\right|$.

Theorem 3.1 and Theorem 3.3 imply the following.

Corollary 3.4. TARGET SET SELECTION with thresholds at most two is not fpt cost $\rho$-approximable even on bipartite graphs, for any computable function $\rho$, unless $\mathrm{FPT}=\mathrm{W}[\mathrm{P}]$.

\section{Parameterized inapproximability of Max and Min $\boldsymbol{k}$-Influence}

We consider in this section the cardinality constraint maximization and minimization versions of TARGET SET SELECTION.

Theorem 4.1. MAX Closed $k$-Influence and MAX OPEn $k$-InfluenCE are not fpt cost $\rho$-approximable even on bipartite graphs, for any computable function $\rho$, unless FPT $=\mathrm{W}[1]$.

Proof. We provide a fpt gap-reduction with gap $\rho$ from Clique to MAX Closed $k$-Influence. Given an instance $I=(G=(V, E), k)$ of CLIQUE, we construct an instance $I^{\prime}=\left(G^{\prime}=\left(V^{\prime}, E^{\prime}\right)\right.$, thr, $\left.k^{\prime}\right)$ of MAX CLOSED $k$-INFLUENCE as follows. The graph $G^{\prime}$ consists of the incidence graph of $G$ with two vertex sets $V^{\prime \prime}$ and $E^{\prime \prime}$ corresponding to the vertices of $G$ and the edges of $G$, as well as a set $Z$ of $h(k)\left(\begin{array}{l}k \\ 2\end{array}\right)$ new vertices $z_{1}^{1}, \ldots, z_{\left(\begin{array}{c}k \\ 2\end{array}\right)}^{1}, \ldots, z_{1}^{h(k)}, \ldots, z_{\left(\begin{array}{c}k \\ 2\end{array}\right)}^{h(k)}$ (the function $h$ will be determined later). For $i=1, \ldots,\left(\begin{array}{c}k \\ 2\end{array}\right)$, there is an edge directed 


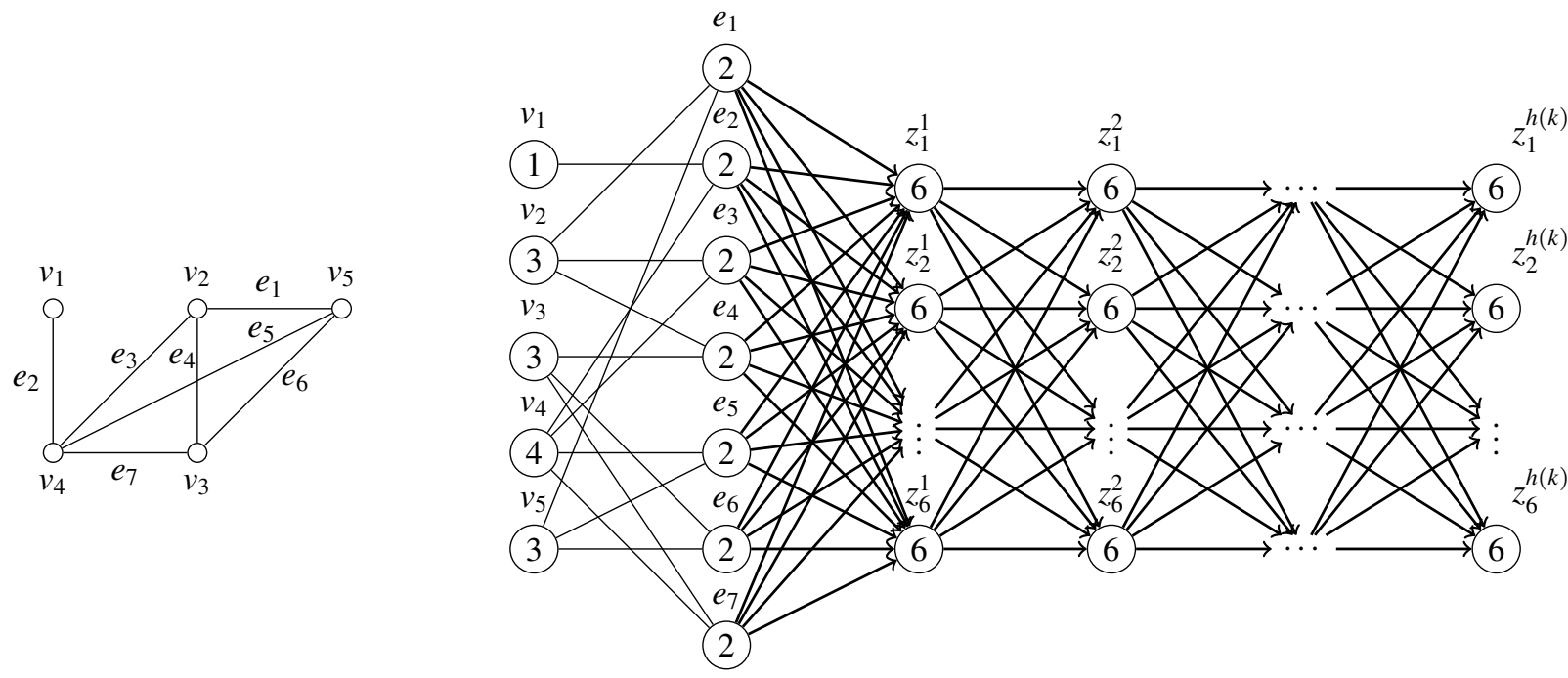

Figure 4. Illustration of the reduction from CLIQUE described in Theorem 4.1 with $k=4$. All arrows on the right graph are directed edge gadgets. Thresholds are represented inside each vertex.

gadget between any vertex of $E^{\prime \prime}$ and vertex $z_{i}^{1}$. Moreover, for any $i=1, \ldots, h(k)-1$, there is an edge directed gadget between $z_{j}^{i}$ and $z_{t}^{i+1}$, for any $j, t \in\left\{1, \ldots,\left(\begin{array}{l}k \\ 2\end{array}\right)\right\}$. We set $k^{\prime}=k$ and $g(k)=k+\left(\begin{array}{l}k \\ 2\end{array}\right)+4\left(\begin{array}{l}k \\ 2\end{array}\right)^{2}$ (recall that there are 4 vertices per directed edge gadget). We finally set the threshold function as follows: $\operatorname{thr}(v)=\operatorname{deg}_{G}(v)$ for all vertices $v \in V^{\prime \prime}, \operatorname{thr}(v)=2$ for all vertices $v \in E^{\prime \prime}$ and $\operatorname{thr}(v)=\left(\begin{array}{l}k \\ 2\end{array}\right)$ for all vertices $v \in Z$. Let $x$ be the smallest integer such that $x / \rho(x) \geq g(k)$. Note that such a $x$ exists and can be computed in time depending only on $k$. We choose $h$ such that $h(k)$ is an integer and $k+(h(k)+1)\left(\begin{array}{l}k \\ 2\end{array}\right)+4 h(k)\left(\begin{array}{l}k \\ 2\end{array}\right)^{2} \geq x$ (see Figure 4).

If $G$ contains a clique of size $k$, then, by activating the same $k$ vertices in $V^{\prime \prime}$, we activate in the next step exactly $\left(\begin{array}{l}k \\ 2\end{array}\right)$ vertices in $E^{\prime \prime}$ (the vertices correspond to the edges of the clique). During the next steps, all the vertices of $Z$ are activated. Overall, opt $\left(I^{\prime}\right) \geq k+(h(k)+1)\left(\begin{array}{l}k \\ 2\end{array}\right)+4 h(k)\left(\begin{array}{l}k \\ 2\end{array}\right)^{2} \geq x$. Moreover, $g(k) \leq x / \rho(x) \leq \operatorname{opt}\left(I^{\prime}\right) / \rho\left(\operatorname{opt}\left(I^{\prime}\right)\right)$, since the function $t / \rho(t)$ is nondecreasing. Otherwise, suppose that there is no clique of size $k$ in $G$. Without loss of generality, we may assume that $k \geq 4$ and thus $k<\left(\begin{array}{l}k \\ 2\end{array}\right)$. Remark that we may also assume that no vertex from $Z$ is in an optimal solution since it activates only $4\left(\begin{array}{l}k \\ 2\end{array}\right)$ more vertices from gadgets and since the threshold of vertices of $Z$ is $\left(\begin{array}{l}k \\ 2\end{array}\right)$, no more vertex from $Z$ can be activated. From the same reason, no vertex from the edge directed gadgets is in an optimal solution. Thus, only vertices from $V^{\prime \prime} \cup E^{\prime \prime}$ are in the optimal solution. Suppose that $t \geq 0$ vertices $S_{1}=\left\{e_{1}^{\prime}, \ldots, e_{t}^{\prime}\right\}$ are activated in $E^{\prime \prime}$ and $k-t$ vertices $S_{2}=\left\{v_{1}^{\prime}, \ldots, v_{k-t}^{\prime}\right\}$ in $V^{\prime \prime}$. Then there is no way to activate $\left(\begin{array}{l}k \\ 2\end{array}\right)$ vertices in $E^{\prime \prime}$. Assume by contradiction that this is possible. If $t=0$ then $\left|\mathcal{A}_{G^{\prime}, \text { thr }}^{1}(S) \cap E^{\prime \prime}\right|<\left(\begin{array}{l}k \\ 2\end{array}\right)$ since there is no clique of size $k$ in $G$, a contradiction. If $t=k$ then $\left|\mathcal{A}_{G^{\prime}, \text { thr }}^{1}(S) \cap E^{\prime \prime}\right| \leq k<\left(\begin{array}{l}k \\ 2\end{array}\right)$ by assumption, a contradiction. Thus $1 \leq t<k$ and then $\left|\mathcal{A}_{G^{\prime}, \text { thr }}^{1}(S) \cap E^{\prime \prime}\right| \leq\left(\begin{array}{c}k-t \\ 2\end{array}\right)+t<\left(\begin{array}{c}k \\ 2\end{array}\right)$, a contradiction. Thus opt $\left(I^{\prime}\right)<k+$ $\left(\begin{array}{l}k \\ 2\end{array}\right)+4\left(\begin{array}{l}k \\ 2\end{array}\right)^{2}=g(k)$. The result follows from Theorem 2.3 together with the W[1]-hardness of CLIQUE [12].

The same reduction works for MAX OPEN $k$-INFLUENCE except that $g(k)=\left(\begin{array}{l}k \\ 2\end{array}\right)+4\left(\begin{array}{l}k \\ 2\end{array}\right)^{2}$ and that $h$ is defined such that $(h(k)+1)\left(\begin{array}{l}k \\ 2\end{array}\right)+4 h(k)\left(\begin{array}{l}k \\ 2\end{array}\right)^{2} \geq x$.

We remark that the proof of Theorem 4.1 shows a stronger result: Unless FPT $=$ W[1], there is no fpt cost $\rho$ approximation for MAX CLOSED $k$-INFLUENCE and MAX OPEN $k$-INFLUENCE, for any computable function $\rho$, even if the running time is of the form $f(k, \ell) \cdot n^{O(1)}$. Here $\ell$ is the cost-parameter passed as an argument to the algorithm, that is, $\ell$ indicates the number of activated vertices. 
As the reductions behind Corollaries 3.2 and 3.4 are not fpt gap-reductions, we cannot use them to prove the same cost inapproximability results for MAX CLOSED $k$-INFLUENCE or MAX OPEN $k$-INFLUENCE with majority thresholds and thresholds at most two.

Minimization variants. In this following, we study minimization versions of the problem. One practical application could be in security of social networks, for example, finding the $k$ most trustworthy individuals such that the leak of critical information is minimized.

In contrast to the maximization versions which remain NP-hard for unanimity thresholds [3], we can show that the minimization variants are polynomial-time solvable for unanimity thresholds.

Proposition 4.2. Min OPEn $k$-INFLUEnCE and Min Closed $k$-InfluenCE are solvable in polynomial time for unanimity thresholds.

Proof. Consider any instance of Min Open $k$-Influence. We have opt $=0$ or 1 and opt $=1$ if and only if $k=$ $n-1$. In order to see this, we distinguish two cases depending on the connectivity of $G$. If $G$ is connected, let $T$ be a spanning tree of $G$. An optimal solution $S$ is constructed by adding iteratively a vertex $v$ in $S$ such that $T-\{v\}$ remains a spanning tree of $G-\{v\}$. If $k<n-1$, then any vertex in $V \backslash S$ has a neighbor in $V \backslash S$ and so $o p t=0$. If $G$ is not connected and $k<n-1$, a solution of value 0 always exists and consists of all the vertices of a connected component or all the vertices of a connected component except at least two connected vertices. Clearly, Min CLOSED $k$-INFLUENCE is also solvable in polynomial time.

The next result shows that Min Closed $k$-InfluenCE and Min OPEN $k$-INFLUENCE are also computationally hard even for thresholds bounded by two. To this end, we consider the decision version of MIN CLOSED $k$ INFLUENCE (resp. Min OPEn $k$-INFLUENCE) denoted by ClOSED $k$-INFLUENCE $\leq$ (resp. OPEN $k$-INFLUENCE $\leq$ ) and defined as follows: Given a graph $G=(V, E)$, a threshold function thr: $V \rightarrow \mathbb{N}$, and integers $k$ and $\ell$, determine whether there is a subset $S \subseteq V,|S|=k$ such that $\left|\mathcal{A}_{G \text {,thr }}(S)\right| \leq \ell\left(\right.$ resp. $\left.\left|\mathcal{A}_{G, \text { thr }}(S) \backslash S\right| \leq \ell\right)$.

Theorem 4.3. CLOSED $k$-INFLUENCE $\leq$ is W[1]-hard w.r.t. parameter $(k, \ell)$ even for threshold bounded by two and bipartite graphs. OPEN $k$-INFLUENCE $\leq$ is NP-hard even for threshold bounded by two, bipartite graphs and $\ell=0$.

Proof. We provide a parameterized reduction from the W[1]-hard problem IndePEndent SET to CLOSED $k$-INFLUENCE $\leq$. Given an instance $(G=(V, E), k)$ of INDEPENDENT SET, we construct an instance $\left(G^{\prime}=\right.$ $\left.\left(V^{\prime}, E^{\prime}\right), k^{\prime}, \ell^{\prime}\right)$ of CLOSED $k$-INFLUENCE $\leq$ by considering the incidence graph. Let $G^{\prime}$ be the bipartite graph with two vertex sets $V$ and $E$ and for each edge $e=u v \in E$, we add $u e, v e \in E^{\prime}$. We define $\operatorname{thr}(u)=1, \forall u \in V$ and $\operatorname{thr}(e)=2, \forall e \in E, k^{\prime}=k$ and $\ell^{\prime}=k$. Clearly $G$ contains an independent set of size at least $k$ if and only if $G^{\prime}$ contains a set of vertices $S$ of size $k$ such that $\left|\mathcal{A}_{G, \mathrm{thr}}(S)\right|=k$.

The previous construction also prove that $G$ contains an independent set of size at least $k$ if and only if $G^{\prime}$ contains a set of vertices $S$ of size $k$ such that $\left|\mathcal{A}_{G \text {,thr }}(S) \backslash S\right|=0$, thus proving the NP-hardness of OPEN $k$-INFLUENCE $\leq$ even for $\ell=0$.

We remark that the previous theorem rules out the possibility of any fixed-parameter algorithm with parameter $\ell$ for OPEN $k$-INFLUENCE $<$ assuming $\mathrm{P} \neq \mathrm{NP}$. Moreover, due to its NP-hardness when $\ell=0$, MIN OPEN $k$-INFLUENCE is not at all fpt cost approximable, unless $\mathrm{P}=\mathrm{NP}$.

In the following, we provide a final result regarding fpt cost approximation of MIN CLOSED $k$-INFLUENCE.

Theorem 4.4. Min CLOSED $k$-INFLUENCE with thresholds at most two is not fpt cost $\rho$-approximable even on bipartite graphs, for any computable function $\rho$, unless $\mathrm{FPT}=\mathrm{W}[1]$.

Proof. We provide a fpt gap-reduction with gap $\rho$ from IndePEndent Set to Min Closed $k$-Influence (see also Figure 5). Given an instance $(G=(V, E), k)$ of InDEPENDENT SET, we construct an instance $\left(G^{\prime}=\right.$ $\left.\left(V^{\prime}, E^{\prime}\right), k^{\prime}\right)$ of MIN CLOSED $k$-INFLUENCE by considering the incidence graph, that is $G^{\prime}$ is a bipartite graph with two vertex sets $V$ and $E$ and for each edge $e=u v \in E$, there is $u e, v e \in E^{\prime}$. We define $\operatorname{thr}(u)=1, \forall u \in V$ and 9 

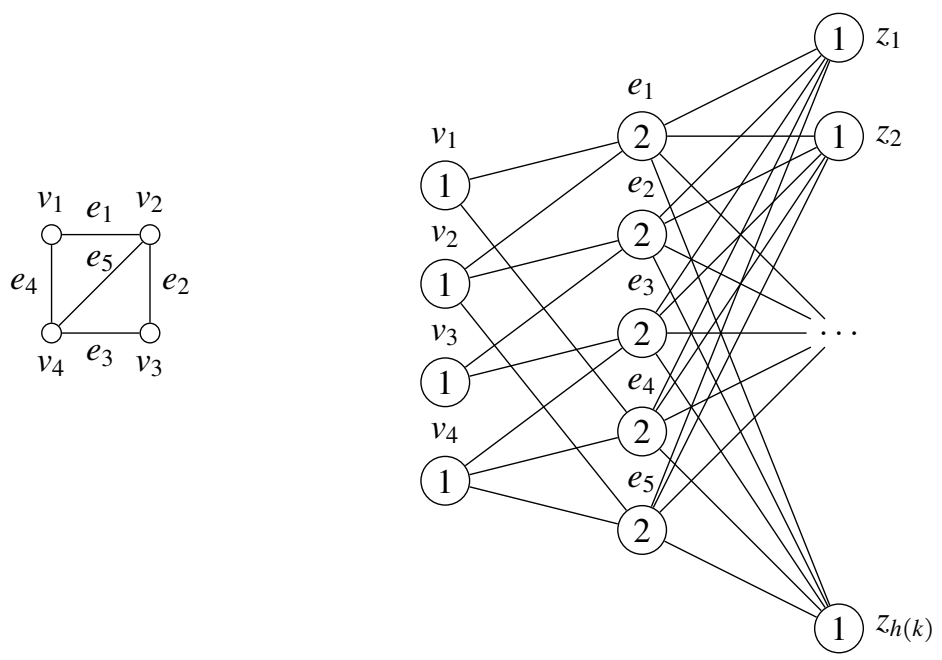

Figure 5. Illustration of the reduction from INDEPENDENT SET as described in Theorem 4.4. Thresholds are represented inside each vertex.

$\operatorname{thr}(e)=2, \forall e \in E$. We choose the function $h$ such that $h(k)$ is an integer and $k+h(k)+1 \geq k \rho(k)$. Then, we add $h(k)$ additional vertices $F$ of threshold 1 in $G^{\prime}$ and a complete bipartite graph between $E$ and $F$. Define $k^{\prime}=k$, $g(k)=k+h(k)+1$. If $G$ contains an independent set of size at least $k$ then, by activating the same $k$ vertices in $G^{\prime}$, we obtain a solution that activates no more vertex in $G^{\prime}$ and thus $\operatorname{opt}\left(I^{\prime}\right)=k \leq \frac{g(k)}{\rho(k)}=\frac{g(k)}{\rho\left(\operatorname{opt}\left(I^{\prime}\right)\right)}$.

If there is no independent set of size $k$, if one activate only two vertices from $F$, it will activate the whole vertex set $E$ on the next step, and then the whole graph. Moreover, activating a vertex from the vertex set $E$ will also activate the whole set $F$ on the next step, and then the whole graph. Finally, activating $k$ vertices of $V$ will activate at least one vertex of $E$ since there is no independent set of size $k$. Note that activating $k-1$ vertices from $V$ and 1 from $F$ will result not be better since vertices of $F$ are connected to all vertices of $E$. Therefore, opt $\left(I^{\prime}\right) \geq k+h(k)+1=g(k)$.

The result follows from Theorem 2.3 together with the W[1]-hardness of INDEPENDENT SET [12].

\section{Conclusion}

Despite the variety of our intractability results, some questions remains open. Are MAX CLOSED $k$-INFLuenCE and MAX OPEN $k$-INFLUENCE fpt cost approximable for constant or majority thresholds? We believe that these problems remain hard, but the classical gadgets used to simulate these thresholds changes does not work for this type of approximation. Similarly, is MIN CLOSED $k$-INFLUENCE fpt cost approximable for majority thresholds?

Finally, the dual problem of TARget Set SELEction (i.e. find a target set of size at most $|V|-k$ ) seems unexplored. Using the fact that TARGET SET SELECTION with unanimity thresholds is exactly Vertex Cover, we know that the dual problem is therefore W[1]-hard, even with unanimity thresholds. But it is still the case for constant or majority thresholds? Moreover, is the dual of TARGET SET SELECTION fpt cost approximable?

\section{References}

[1] Ashkan Aazami and Kael Stilp. Approximation algorithms and hardness for domination with propagation. SIAM J Discrete Math, 23(3):1382-1399, 2009.

[2] Sanjeev Arora and Carsten Lund. Hardness of approximations. In Approximation algorithms for NP-hard problems, pages 399-446. PWS Publishing Company, 1996.

[3] Cristina Bazgan, Morgan Chopin, André Nichterlein, and Florian Sikora. Parameterized approximability of maximizing the spread of influence in networks. J. Discrete Algorithms, 27:54-65, 2014. 
[4] Oren Ben-Zwi, Danny Hermelin, Daniel Lokshtanov, and Ilan Newman. Treewidth governs the complexity of target set selection. Discrete Optim, 8(1):87-96, 2011.

[5] Liming Cai and Xiuzhen Huang. Fixed-parameter approximation: Conceptual framework and approximability results. Algorithmica, 57(2):398-412, 2010.

[6] Ching-Lueh Chang and Yuh-Dauh Lyuu. Spreading messages. Theor Comput Sci, 410(27-29):2714-2724, 2009.

[7] Ning Chen. On the approximability of influence in social networks. SIAM J Discrete Math, 23(3):1400-1415, 2009.

[8] Yijia Chen, Martin Grohe, and Magdalena Grüber. On parameterized approximability. In Hans L. Bodlaender and Michael A. Langston, editors, Proc. of IWPEC '06, volume 4169 of LNCS, pages 109-120. Springer, 2006.

[9] Morgan Chopin, André Nichterlein, Rolf Niedermeier, and Mathias Weller. Constant thresholds can make target set selection tractable. In Guy Even and Dror Rawitz, editors, Proc. of MedAlg '12, volume 7659 of LNCS, pages 120-133. Springer, 2012.

[10] Ferdinando Cicalese, Gennaro Cordasco, Luisa Gargano, Martin Milanic, and Ugo Vaccaro. Latency-bounded target set selection in social networks. Theor. Comput. Sci., 535:1-15, 2014.

[11] Irit Dinur and Shmuel Safra. The importance of being biased. In John H. Reif, editor, Proceedings on 34th Annual ACM Symposium on Theory of Computing (STOC'02), pages 33-42. ACM, 2002.

[12] Rodney G. Downey and Michael R. Fellows. Fundamentals of Parameterized Complexity. Springer, 2013.

[13] Rodney G. Downey, Michael R. Fellows, and Catherine McCartin. Parameterized approximation algorithms. In Hans L. Bodlaender and Michael A. Langston, editors, Proc. of IWPEC '06, volume 4169 of LNCS, pages 121-129. Springer, 2006.

[14] Paul A. Dreyer and Fred S. Roberts. Irreversible $k$-threshold processes: Graph-theoretical threshold models of the spread of disease and of opinion. Discrete Appl Math, 157(7):1615 - 1627, 2009.

[15] Jörg Flum and Martin Grohe. Parameterized Complexity Theory. Springer, 2006.

[16] David Kempe, Jon Kleinberg, and Éva Tardos. Maximizing the spread of influence through a social network. In Lise Getoor, Ted E. Senator, Pedro Domingos, and Christos Faloutsos, editors, Proc. of the 9th SIGKDD International Conference on Knowledge Discovery and Data Mining (KDD ’03), pages 137-146. ACM, 2003.

[17] Daniel Marx. Parameterized complexity and approximation algorithms. Comput J, 51(1):60-78, 2008.

[18] Dániel Marx. Completely inapproximable monotone and antimonotone parameterized problems. J. Comput. Syst. Sci., 79(1):144-151, 2013.

[19] André Nichterlein, Rolf Niedermeier, Johannes Uhlmann, and Mathias Weller. On tractable cases of target set selection. Soc Network Anal Mining, 3(2):233-256, 2013.

[20] Rolf Niedermeier. Invitation to Fixed-Parameter Algorithms. Oxford University Press, 2006.

[21] David Peleg. Local majorities, coalitions and monopolies in graphs: a review. Theor. Comput. Sci., 282(2):231$257,2002$.

[22] T. V. Thirumala Reddy and C. Pandu Rangan. Variants of spreading messages. J Graph Algorithms Appl, 15(5):683-699, 2011. 